\title{
The Expression of Manganese Superoxide Dismutase Gene from Nelumbo nucifera Responds Strongly to Chilling and Oxidative Stresses
}

\author{
Wen $\mathrm{Li}^{1 \dagger}$, Lin $Q \mathrm{i}^{1 \dagger}$, Xiaodong Lin ${ }^{1}$, Huhui Chen ${ }^{1}$, Zhanqi Ma ${ }^{1}$, Keqiang $\mathrm{Wu}^{2}$ and \\ Shangzhi Huang ${ }^{1 *}$ \\ ( ${ }^{1}$ School of Life Sciences, Sun Yat-sen University, Guangzhou 510275, China; \\ ${ }^{2}$ Institute of Plant Biology, School of Life Sciences, National Taiwan University, Taipei 106, China)
}

\begin{abstract}
A manganese superoxide dismutase (Mn-SOD) gene, NnMSD1, was identified from embryonic axes of the sacred lotus (Nelumbo nucifera Gaertn.). The NnMSD1 protein contains all conserved residues of the Mn-SOD protein family, including four consensus metal binding domains and a signal peptide for mitochondrial targeting. Southern blot analysis suggests the existence of two Mn-SOD genes in sacred lotus. NnMSD1 was highly expressed in developing embryonic axes during seed development, but appeared in cotyledons only at the early stage of development and became undetectable in the cotyledons during late embryogenesis. The expression of the NnMSD1 gene in germinating embryonic axes, in response to various stresses such as heat shock, chilling, and exposure to stress-related chemicals, was also studied. Heat shock strongly inhibited the expression of the NnMSD1 gene, whereas the NnMSD1 transcript level increased strongly in chilling stress treatment. An increase in expression was also highly induced by $\mathrm{H}_{2} \mathrm{O}_{2}$ in germinating embryonic axes. The results suggest that the expression pattern of the NnMSD1 gene differed between developing axes and cotyledons, and that the NnMSD1 gene expression responds strongly to chilling and oxidative stress.
\end{abstract}

Key words: manganese superoxide dismutase; sacred lotus (Nelumbo nucifera Gaertn.); seed development and germination; stress.

Li W, Qi L, Lin X, Chen H, Ma Z, Wu K, Huang S (2009). The expression of manganese superoxide dismutase gene from Nelumbo nucifera responds strongly to chilling and oxidative stresses. J. Integr. Plant Biol. 51(3), 279-286.

Available online at www.jipb.net

The sacred lotus (Nelumbo nucifera Gaertn.) is a primitive angiosperm and one of the oldest plants that has survived from the last ice age. Seeds of the sacred lotus hold the world record for seed longevity, with $80 \%$ viability observed in 200-1 300-year-old fruits (Shen-Miller et al. 2002). In order to germinate upon imbibition, an aged seed must be able to quickly repair severe cellular damage accrued over long-term storage.

Received 12 Aug. 2007 Accepted 23 Sep. 2008

Supported by the National Natural Science Foundation of China (30370912) and the Natural Science Foundation of Guangdong Province (04009773 and 2006B20101010).

$\dagger$ These authors contributed equally to this work.

*Author for correspondence.

Tel: +86 2084110797 ;

Fax: +86 208403 6215;

E-mail: <huangsz@mail.sysu.edu.cn>.

(C) 2008 Institute of Botany, the Chinese Academy of Sciences

doi: 10.1111/j.1744-7909.2008.00790.x
The great ability of lotus seeds to survive in extreme stress conditions is also well documented. Lotus seeds maintained $100 \%$ viability even after $24 \mathrm{~h}$ treatment in an air oven at $100{ }^{\circ} \mathrm{C}$ (Huang et al. 2003) and low-dose soil $\gamma$-radiation of $3 \mathrm{~Gy}$ did not affect viability (Shen-Miller et al. 2002).

Superoxide dismutases catalyze the breakdown of superoxide radicals, and provide the first line of defense against oxygen toxicity. They are essential for aerobic life and are involved in stress resistance and longevity (Carlioz and Touati 1986; Orr and Sohal 1994). Three groups of superoxide dismutases (SODs) can be distinguished on the basis of the metal cofactor at the active site: Cu/Zn SOD, Mn-SOD, and Fe-SOD (Fridovich 1989). Mn-SOD and Fe-SOD appear to be closely related both in structural and in evolutionary aspects, but have no resemblance to Cu/ZnSOD (Bowler et al. 1994). Cu/Zn SODs occur in the cytoplasm and chloroplasts; Mn-SODs are usually found in the mitochondrial matrix and in peroxisomes; and Fe-SODs are present within the chloroplasts of some plants (Raychaudhuri and Deng 2000). The roles of SODs under environmental stresses have been studied extensively (Yu and 
Rengel 1999; Raychaudhuri and Deng 2000). It was reported that the Mn-SOD protected Candida albicans against various stresses (Hwang et al. 2003) and the induced overexpressed Mn-SOD extends the lifespan of adult Drosophila melanogaster (Sun et al. 2002).

Unlike most other organisms, plants contain multiple SOD isozymes. Nine SOD isozymes have been described in maize (Baum and Scandalios 1981) and seven in Arabidopsis (Kliebenstein et al. 1999). The first plant SOD gene was cloned from maize (Cannon et al. 1987). Since then, a number of SOD genes have been cloned in other plant species, for example, tobacco (Bowler et al. 1989), tomato (Perl-Treves et al. 1988), rice (Kaminaka et al. 1999), peach (Bagnoli et al. 2002), aspen (Akkapeddi et al. 1994), Arabidopsis (Kliebenstein et al. 1999), camphor tree (Chen et al. 2002), and cassava (Shin et al. 2005). In the present study, we cloned the Mn-SOD gene, NnMSD1 (GenBank Accession No. AY934858), from sacred lotus, and investigated its expression during seed development and germination, as well as its responses to various stresses.

\section{Results}

\section{Structure of the sacred lotus NnMSD1 gene}

The full length cDNA of NnMSD1 contains an open reading frame (ORF) of 699 nucleotides, flanked by 35 bp of a noncoding sequence at the $5^{\prime}$ end and $251 \mathrm{bp}$ of a non-coding sequence with a $21 \mathrm{bp}$ poly $(\mathrm{A})$ tail at the $3^{\prime}$ end (Figure 1). Kozak consensus sequence was identified in the $5^{\prime}$-non-coding sequence, which may facilitate the initial binding of mRNA to the small submit of the ribosome. A comparison with the sequence of the NnMSD1 gene and its cDNA sequence indicated that six exons and five introns were present in the gene. The introns have a high AT content (>60\%). The Intron 2 and Intron 3 are $102 \mathrm{bp}$ and $142 \mathrm{bp}$, respectively, which are much shorter than the other three Introns (Table 1). The cDNA of NnMSD1 encodes a protein of 233 amino acid residues with a predicted molecular mass of $25.9 \mathrm{kDa}$ and an isoelectric point of 6.6 .

\section{Comparison of the amino acid sequences of Mn-SOD genes from different species and analysis of conserved residues}

Alignment of the deduced amino acid sequence of $\mathrm{Mn}$ SOD from sacred lotus (AAX22235) with those from the other plants indicates that the $\mathrm{N}$-terminal regions are less conserved (Figure 2). The sequences of $\mathrm{Mn}$-SOD from Triticum aestivum (AAX68501), Pisum sativum (CAA42737), Zea mays (AAA72020), Oryza sativa (Q43008), Camellia sinensis (AAT68778), Prunus persica (CAB56851), Arabidopsis thaliana (AAC24832), Nicotiana plumbaginifolia (CAA32643) and sacred lotus share similarities that range from $73 \%$ to
$78 \%$. In addition, the sequences of Fe-SOD from $P$. sativum (CAD42655), Z. mays (BAD89495), A. thaliana (NP849441), O. sativa (XP550626), Lycopersicon esculentum (AAQ18699), Glycine max (AAQ13492), Vigna unguiculata (AAF28773) and $\mathrm{Mn}-\mathrm{SOD}$ from sacred lotus share similarities that range from $25 \%$ to $30 \%$.

Although plant Mn-SOD N-terminal regions are poorly conserved, they have the general features of mitochondrial targeting sequences, which direct Mn-SOD proteins to the mitochondrion. The N-terminal sequence of NnMSD1 shares common characteristics with mitochondrial transit peptides, such as an abundance of basic residues and the complete absence of acidic residues. Based on signal PV2.0 (Nielsen et al. 1997) and TARGET PV1.0 (Emanuelsson et al. 2000) prediction to $\mathrm{N}$-terminal peptides, the deduced sacred lotus peptide has an $\mathrm{N}$-terminal signal peptide of 24 amino acids (MALRSVISRKTLGSLGLGFSHARG) for mitochondrial subcellular localization. The Mn-SOD sequences contain four perfectly conserved regions in all plant species, namely consensus I to IV (Figure 1). Homology is most conserved in the domains corresponding to $\alpha$ helices one (consensus V) and two (consensus III). In addition, the amino acids involved in the coordination of the metal ions are highly conserved (Borgstahl et al. 1992). After removing the 24 amino acid signal peptides, the predicted mature sacred lotus Mn-SOD and the other plant Mn-SODs share sequence similarities ranging from $77 \%$ to $81 \%$. The predicted mature NnMSD1 showed highest homology (81\%) to the Mn-SODs from $C$. sinensis, $P$. persica, and $N$. plumbaginifolia. The mature sacred lotus Mn-SOD protein has a calculated molecular mass of approximately $23.4 \mathrm{kDa}$ and a pl of 5.9 .

Phylogenetic analysis of Mn-SODs from different plant species revealed two subgroups (Figure 2). It should be noted that all Mn-SODs from cereals are grouped in the same subgroup. Subgroup 1 consists of the sequences from $P$. sativum, C. sinensis, A. thaliana, P. persica, N. plumbaginifolia, N. nucifera, Avicennia marina, Capsicum annuum, Euphorbia esula, and Zantedeschia aethiopica.

\section{Genomic organization of NnMSD1 gene}

In order to determine the copy number of the NnMSD1 gene, the genomic DNA $(5 \mu \mathrm{g})$ of sacred lotus leaves was digested with EcoRI, HindIII, or Bg/lI and hybridized with a 423 bp ${ }^{32} \mathrm{P}$-labeled probe. Two strongly hybridizing bands were detected for each restriction enzyme (Figure 3). These data indicate that there are two NnMSD1 genes in the sacred lotus genome.

\section{Expression of NnMSD1 gene in developing and germinating seeds}

The development pattern of sacred lotus seeds is similar to that of most dicot seeds. There are three stages in the sacred lotus seed development: tissue differentiation (0-13 d after 


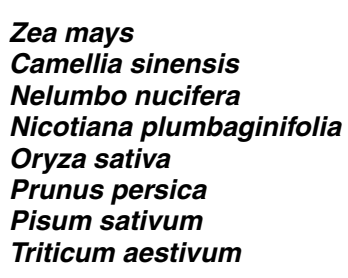

Z. mays

C. sinensis

$N$. nucifera

N. plumbaginifolia

O. sativa

P. persica

$P$. sativum

T. aestivum

Z. mays
C. sinensis
N. nucifera
N. plumbaginifolia
O. sativa
P. persica
P. sativum
T. aestivum

Z. mays

C. sinensis

$N$. nucifera

N. plumbaginifolia

O. sativa

$P$. persica

P. sativum

T. aestivum

Z. mays
C. sinensis
N. nucifera
N. plumbaginifolia
O. sativa
P. persica
P. sativum
T. aestivum
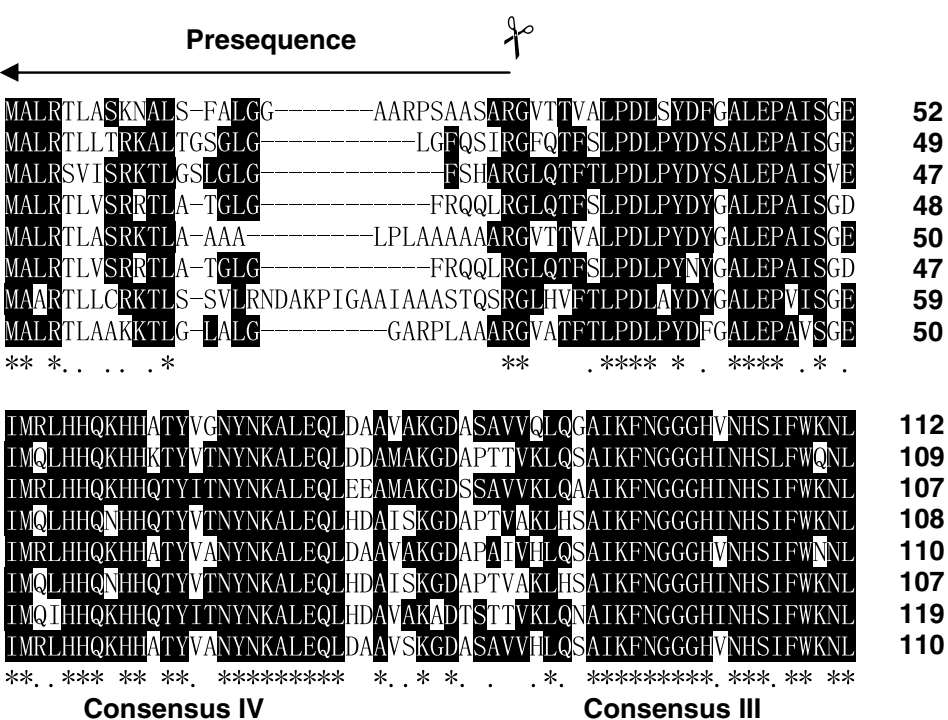

112

109

107

108

110

107

119

110
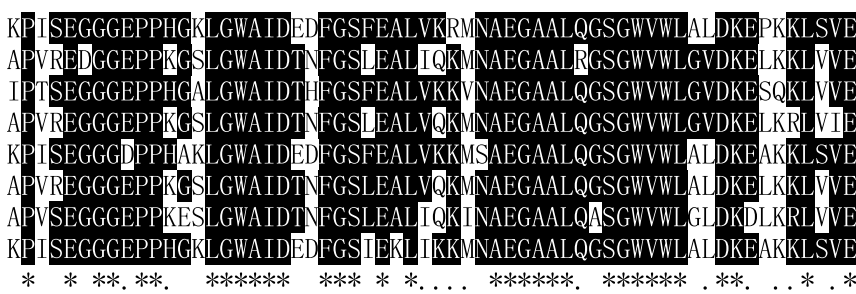

172

169

167

168

170

167

179

170

Consensus II

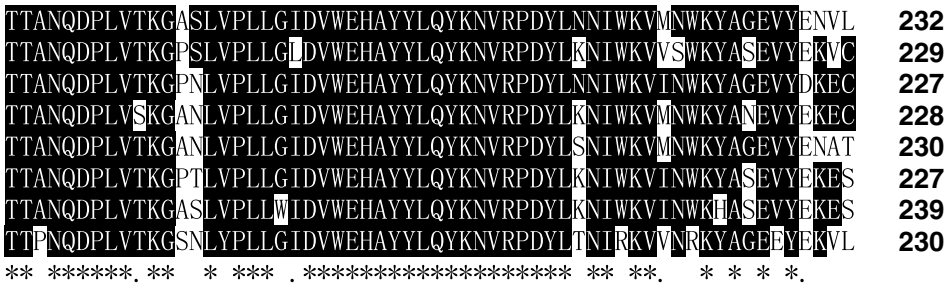

Consensus I

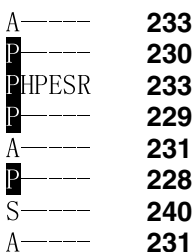

Figure 1. Alignment of the NnMSD1 deduced polypeptide sequence with other manganese superoxide dismutases (Mn-SODs) from various plant species.

The amino acid residues conserved in sacred lotus and the other plants are marked by white letters in a black box. The listed Mn-SODs are from Triticum aestivum (AAX68501), Pisum sativum (CAA42737), Zea mays (AAA72020), Oryza sativa (Q43008), Camellia sinensis (AAT68778), Prunus persica (CAB56851), Nelumbo nucifera (AAX22235) and Nicotiana plumbaginifolia (CAA32643).

pollination, DAP), cell expansion (14-20 DAP) and desiccationmaturation stage (21-30 DAP). The expression patterns of the NnMSD1 gene were investigated during seed development by reverse transcriptase-polymerase chain reaction (RT-PCR)
(Figure 4A). Strong and constant levels of NnMSD1 expression were detected in sacred lotus embryonic axes during seed development. The cotyledons showed low NnMSD1 gene expression on the 15 DAP, whereas there was no detectable 
Table 1. Size, percent $A+T$ and sequence characteristics of the five introns of the sacred lotus NnMSD1 gene

\begin{tabular}{llccc}
\hline Intron & Intron/exon boundaries & Size (bp) & $\mathrm{A}+\mathrm{T}(\%)$ & $\begin{array}{c}\text { Nucleotide } \\
\text { position }\end{array}$ \\
\hline 1 & AG/gtaatt. ..tgcag/GG & 772 & 65.2 & $322-1094$ \\
2 & AGT/gtaagt. ..tgcag/GA & 104 & 72.1 & $1141-1244$ \\
3 & TG/gtaagt. . .ggcag/TG & 142 & 66.9 & $1371-1512$ \\
4 & AG/gtgaagt. ..tacag/GA & 924 & 66.3 & $1570-2493$ \\
5 & CAG/gtgag. ..tgcag/TA & 723 & 62.8 & $2572-3294$ \\
\hline
\end{tabular}

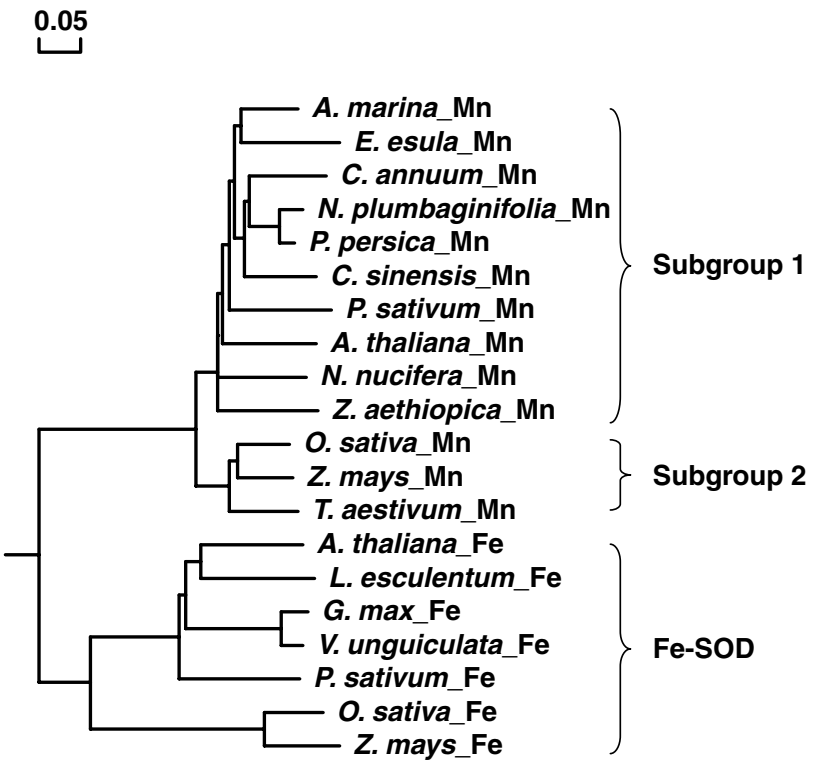

Figure 2. Phylogenetic relationship of the deduced amino acid sequences of manganese superoxide dismutase (Mn-SOD) and Fe-SOD families from different plant species.

Cluster analysis was carried out using the DNAMAN Alignment Program. The species and corresponding accession numbers are as follows. Mn-SOD: Triticum aestivum (AAX68501), Pisum sativum (CAA42737), Zea mays (AAA72020), Oryza sativa (Q43008), Camellia sinensis (AAT68778), Capsicum annuum (AAB88870), Avicennia marina (AAN15216), Euphorbia esula (AF242310), Zantedeschia aethiopica (AF094832), Prunus persica (CAB56851), Nelumbo nucifera (AAX22235), N. plumbaginifolia (CAA32643) and A. thaliana (AAC24832); Fe-SOD: P. sativum (CAD42655), Z. mays (BAD89495), A. thaliana (NP-849441), O. sativa (XP-550626), Lycopersicon esculentum (AAQ18699), Glycine max (AAQ13492), and Vigna unguiculata (AAF28773).

NnMSD1 transcript in the cotyledons of 20 DAP and 25 DAP (Figure 4A).

The expression profile of the NnMSD1 gene during sacred lotus seed germination was examined using gene-specific primers (Figure 4B). NnMSD1 transcript was detected in mature dry embryonic axes. Within $24 \mathrm{~h}$ after imbibition, the transcript levels

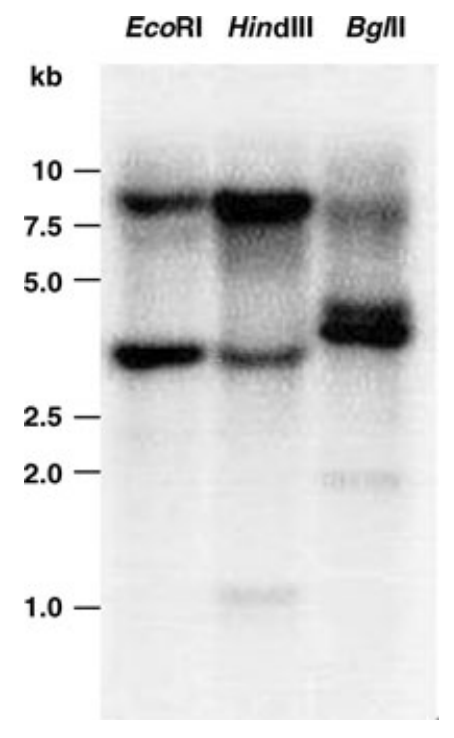

Figure 3. Southern blot of genomic DNA from sacred lotus leaves.

Five micrograms of genomic DNA was digested with the restriction endonucleases (EcoRI, HindIII, and Bg/II), separated by agarose gel and transferred onto a piece of nylon membrane (Hybond- $\mathrm{N}^{+}$, Amersham Biosciences). The blotted membrane was hybridized with a $\left[{ }^{32} \mathrm{P}\right]$-labeled probe from NnMSD1.

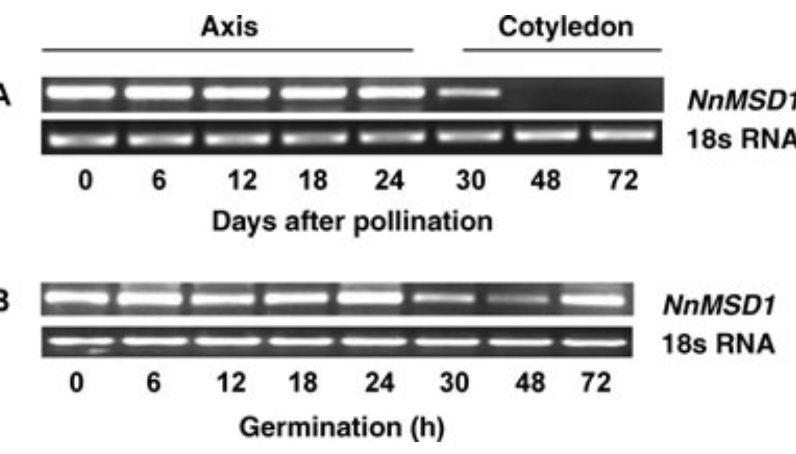

Figure 4. Expression of NnMSD1 gene in developing and germinating embryonic axes and cotyledons of sacred lotus by reverse transcriptionpolymerase chain reaction (RT-PCR).

(A) Expression pattern of NnMSD1 gene in developing sacred lotus axes and cotyledons.

(B) Expression profile of the NnMSD1 gene in germinating sacred lotus axes.

18s RNA was used as a control for equal loading.

of the NnMSD1 gene in sacred lotus embryonic axes were the same as in the dry embryonic axes. However, its expression decreased obviously in the $30 \mathrm{~h}$ and $48 \mathrm{~h}$ after imbibition, and was restored in the $72 \mathrm{~h}$ after imbibition (Figure 4B). 


\section{Expression patterns of the NnMSD1 gene in germinated embryonic axes under various stress conditions}

The expression patterns of the NnMSD1 gene in germinated sacred lotus embryonic axes under various stresses, such as heat shock $\left(42^{\circ} \mathrm{C}\right)$, chilling $\left(4^{\circ} \mathrm{C}\right)$, and exposure to stressrelated chemicals, were investigated by RT-PCR. The stressrelated chemicals include polyethylene glycol (PEG)-6000 (20\% w/v), $\mathrm{H}_{2} \mathrm{O}_{2}(1.5 \%), \mathrm{NaCl}(300 \mathrm{mmol} / \mathrm{L})$, and abscisic acid (ABA) $(100 \mu \mathrm{mol} / \mathrm{L})$. The results of RT-PCR analysis show that NnMSD1 in the germinated embryonic axes responded strongly to chilling and oxidative stresses. However, NnMSD1 gene expression in germinated embryonic axes was inhibited by heat-shock and $\mathrm{NaCl}$. No obvious effects of $\mathrm{ABA}$ and PEG on the NnMSD1 gene expression were observed (data not shown).

To get more information about the expression of NnMSD1 under chilling and oxidative stresses, real-time PCR was used to study the transcription of NnMSD1 after chilling and $1.5 \%$ of $\mathrm{H}_{2} \mathrm{O}_{2}$ treatments, respectively. For studying the effects of chilling on the NnMSD1 mRNA levels, 3-d-old germinated sacred lotus seeds were treated with chilling $\left(4^{\circ} \mathrm{C}\right)$ for 2,6 , 12, 24 and $48 \mathrm{~h}$, respectively. NnMSD1 was strongly expressed after 2-48 $\mathrm{h}$ chilling treatment (Figure 5A).

To study NnMSD1 gene response to $\mathrm{H}_{2} \mathrm{O}_{2}$, the 3-d-old germinated seeds were incubated separately in aqueous solutions containing $1.5 \%$ of $\mathrm{H}_{2} \mathrm{O}_{2}$ for $2,6,12,24$ and $48 \mathrm{~h}$, respectively. The results suggested that the expression of NnMSD1 in the germinated embryonic axes was inhibited after 2, 6 and $48 \mathrm{~h}$ treatment, but stimulated significantly after 12 and $24 \mathrm{~h}$ treatment (Figure 5B).

\section{Discussion}

We have isolated and characterized the NnMSD1 gene encoding a Mn-SOD from sacred lotus embryonic axes. NnMSD1 has six exons and five introns, which is consistent with the MnSOD genes identified from other plant species (Fink and Scandalios 2002). The deduced amino acid sequence of NnMSD1 revealed a high degree of homology with Mn-SODs from other plants (Figure 1). Sacred lotus mature Mn-SOD protein displays high homology (81\%) to the Mn-SOD proteins from C. sinensis, $P$. persica, and N. plumbaginifolia. The 24-amino acid stretch located upstream of the immature Mn-SOD protein could be the leader sequence for translocation into the mitochondrial matrix (Schatz and Krebs 1987). Two copies of the MnSOD gene are present in the sacred lotus genome, which is similar to the peach MnSOD gene (Bagnoli et al. 2002), whereas in maize and wheat the corresponding genes have been reported to belong to small multigene families (Zhu and Scandalios 1994; Wu et al. 1999). The existence of two sacred lotus MnSOD copies could provide an explanation for the variation in the number of Mn-SOD isoforms found in the cotyledons (Huang
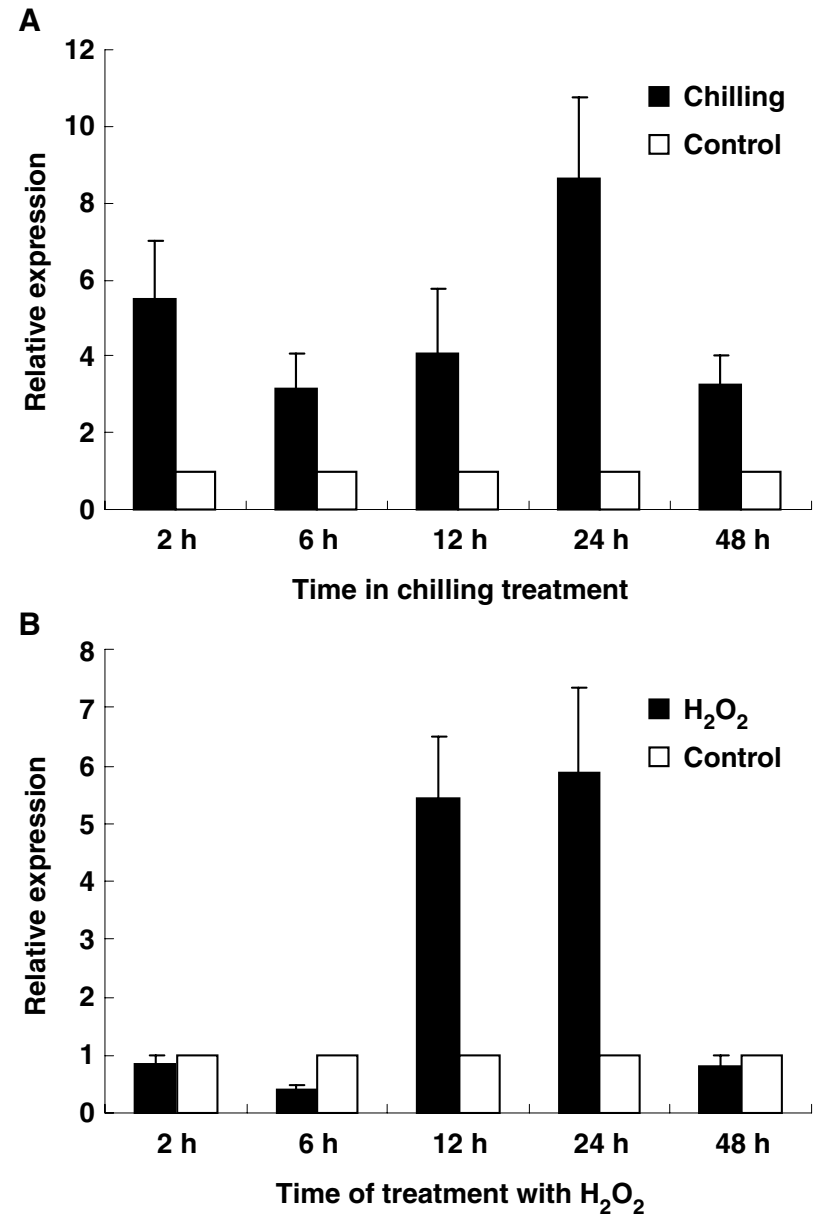

Figure 5. Real-time polymerase chain reaction (PCR) analysis of $\mathrm{Nn}$ MSD1 expression in germinated sacred lotus embryonic axes under chilling and $\mathrm{H}_{2} \mathrm{O}_{2}$ stress.
(A) Chilling.
(B) $\mathrm{H}_{2} \mathrm{O}_{2}$ treatment.

et al. 2000) and the other different tissues analyzed (data not shown). In contrast with our results, Ushimaru et al. (2001) reported that seven electrophoretically distinct SOD isozymes, two Fe-SOD and five Cu/Zn-SOD isozymes, were detectable in sacred lotus seedlings. No Mn-SOD isozyme was detected in their experimental system.

The roles of SODs in plants under environmental stresses have been studied extensively. It was reported that the MnSOD protected Candida albicans against various stresses (Raychaudhuri and Deng 2000). Plants often experience temperature stress during their life cycle (Stone 2001). The expressions of $\mathrm{Mn}-\mathrm{SOD}$ genes under temperature stress were studied extensively in plants. The Mn-SOD gene was induced in both winter and spring cereals that were subjected to cold-acclimating conditions (Wu et al. 1999). However, Tsang et al. (1991) found 
that the expression of Mn-SOD gene in tobacco was unaffected by chilling but that SOD gene expression increased rapidly after the plants were returned to their normal growth temperature. It was reported that transgenic alfalfa overexpressing Mn-SOD had enhanced freezing-stress tolerance (McKersie et al. 1993). The presence of transgenic Mn-SOD had clear effects on maize foliar tolerance to chilling (Breusegem et al. 1999). Our results indicated that the chilling caused an increase in the NnMSD gene expression in $48 \mathrm{~h}$ treated period (Figure $5 \mathrm{~A}$ ). However, heat shock always induces the expression of the MnSOD gene (Privalle and Fridovich 1987). In the germinating sacred lotus seeds, heat shock significantly inhibited NnMSD1 gene expression. Considering that the breakdown of superoxide radicals may produce excessive $\mathrm{H}_{2} \mathrm{O}_{2}$, which will do harm to the cell, the inhibition can be protective to the germinating seeds.

When seedlings were treated with $\mathrm{NaCl}$, the content of $\mathrm{H}_{2} \mathrm{O}_{2}$ was elevated. At the same time, the expression of Mn-SOD was preferentially elevated by salt stress in rice and mangrove (Fadzilla et al. 1997; Lee et al. 2001; Parida et al. 2004). Furthermore, with long term $\mathrm{NaCl}$ treatments, the transcript level for mitochondrial Mn-SOD was strongly induced in the $\mathrm{NaCl}$ tolerant variety of pea, but not in the $\mathrm{NaCl}$-sensitive variety (Hernández et al. 2000). It strongly suggested that oxidative stress was induced by salt stress and Mn-SOD play an important role in the response of plants to salt stress. This was proved by the fact that transgenic Arabidopsis overexpressing Mn-SOD enhanced salt-tolerance (Wang et al. 2004). Our results showed that the oxidative stress $\left(\mathrm{H}_{2} \mathrm{O}_{2}\right)$ treatment could increase the expression of the NnMSD1 gene in sacred lotus germinating axes (Figure 5B). However, the expression of the NnMSD1 gene was inhibited completely by $300 \mathrm{mmol} / \mathrm{L} \mathrm{NaCl}$ treatment. Therefore, the variety of early germinating sacred lotus seeds was argued to be sensitive to strong salt stress. Since maize Sod3. 1 does not respond to ABA, whereas the expression maize Sod3.2, Sod3.3 and Sod3.4 are induced by ABA (Zhu and Scandalios 1994), it was possible that different transcripts of Mn-SOD genes showed various responses to ABA.

Since overexpressing Mn-SOD extends the lifespan of adult Drosophila melanogaster (Sun et al. 2002), it was intriguing to study the function of the NnMSD1 gene on sacred lotus seed longevity. In our previous study, we demonstrated that the sacred lotus embryonic axes contained more than four isoforms of Fe-SOD and one Mn-SOD (Huang et al. 2000). In addition, the total activity of SOD in sacred lotus developing embryonic axes slightly increased from 6 DAP to 20 DAP, then increased sharply and reached its maximum at $25 \mathrm{DAP}$, and this high activity of SOD was retained to complete maturation of the seed (Huang et al. 2003). The results in this paper showed that strong and constant expression of the NnMSD1 gene was detected in sacred lotus embryonic axes during seed development and maturation. Furthermore, the abundant NnMSD1 transcripts also existed in the axes of mature and dry seeds (Figure 4A).
Our studies suggest a possible role of Mn-SOD in sacred lotus seed tolerance against environmental stresses. The function of sacred lotus NnMSD1 gene in seed thermotolerance will be studied further by developing transgenic plant lines.

\section{Materials and Methods}

\section{Plant materials and treatments with exogenous factors}

Seeds of sacred lotus (Nelumbo nucifera cv. Hongtoujin) were collected from plants grown in ponds in Sanshui Lotus World, Guangdong Province, China. Developing embryonic axes were used for gene cloning and RNA preparation in the study of gene expression during seed development. Mature seeds were used in germination test. Seeds were germinated in distilled water at $25^{\circ} \mathrm{C}$ for $0,1,2$, and $3 \mathrm{~d}$. Seeds that germinated for $3 \mathrm{~d}$ were subjected to various stress treatments by submerging in aqueous solutions containing PEG 6000 (20\% w/v), $\mathrm{H}_{2} \mathrm{O}_{2}$ (1.5\%), $\mathrm{NaCl}(300 \mathrm{mmol} / \mathrm{L})$, and $\mathrm{ABA}(100 \mu \mathrm{mol} / \mathrm{L})$ for $24 \mathrm{~h}$ or incubating at different temperatures (heat shock at $42^{\circ} \mathrm{C}$ and low temperature at $4^{\circ} \mathrm{C}$ ) for up to $48 \mathrm{~h}$. All treated materials were immediately frozen in liquid nitrogen and stored at $-70^{\circ} \mathrm{C}$ until use.

\section{RNA preparation and RACE}

Total RNA was isolated from developing embryonic axes by using Trizol Reagent (Invitrogen, Carlsbad, CA, USA), according to the manufacturer's instructions. Adapter ligated cDNA was synthesized using the SMART rapid amplification of cDNA ends (RACE) cDNA amplification kit (BD Biosciences, San Jose, CA, USA) according to the product manual. 5'-RACE was carried out using $5^{\prime}$ primer and the degenerate primer (DP: 5'-AGGTAGTAA/CGCATGCTCCCA-3') designed from the conserved region of the plant SODs. 3'-RACE was carried out using CDSIII /3' PCR primer and the gene specific primer (GSP1: 5'-TTGAAGCATTGGTGAAAAAGG-3') designed according to sequence of the $680 \mathrm{bp}$ fragment. PCR products were purified and sequenced. As the first strand cDNA was synthesized with the SMART cDNA synthesis kit, only one gene-specific primer was used for $5^{\prime}$ RACE based on the conserved domain WEHAYY of Mn-SOD. The 5' end RACE product was recovered and sequenced directly. A gene-specific primer was designed for $3^{\prime}$ RACE according to the acquired $5^{\prime}$ fragment of the NnMSD1 gene from sacred lotus cDNA. After the two sequences were assembled, the full length cDNA of NnMSD1 was obtained.

\section{DNA and PCR amplification}

Genomic DNA was extracted from sacred lotus leaves according to the protocol described by Pich and Schubert 
(1993). Two gene-specific primers GSP2 (5'-GGCGCTGAT CAAATTGGAAG-3') and GSP3 (5'-GAAGACTGCCTGCCC AACTC- $3^{\prime}$ ) were designed based on the sequence of the acquired NnMSD1 cDNA to amplify the corresponding genomic DNA sequence. The PCR product of 3469 bp was sequenced directly.

\section{Analysis of the DNA sequence}

The homologous sequences of the gene NnMSD1 were searched using BLAST (Altschul et al. 1990) and the sequence alignments were carried out using the Cluster $\mathrm{W}$ program (Thompson et al. 1994). The intron position of NnMSD1 gene was determined by comparison of the genomic DNA sequence with the corresponding cDNA sequence.

\section{Southern blot analysis}

Five micrograms of genomic DNA was digested with the restriction endonucleases EcoRI, HindIII, and $\mathrm{Bg} / \mathrm{II}$ at $37^{\circ} \mathrm{C}$ overnight, and the resulting fragments were resolved in a $0.8 \%$ agarose gel and transferred onto a piece of nylon membrane (Hybond- ${ }^{+}$, Amersham Biosciences, Uppsala, Sweden). The blotted membrane was UV cross-linked and hybridized with a [32P]-labeled probe from NnMSD1 (position 1281-1703). In the high stringency condition, the membrane was hybridized at $42{ }^{\circ} \mathrm{C}$ for $14 \mathrm{~h}$ and washed twice with $0.1 \%$ sodium dodecyl sulfate (SDS) in $2 \times$ standard saline citrate (SSC) $(150 \mathrm{mmol} / \mathrm{L}$ $\mathrm{NaCl}, 15 \mathrm{mmol} / \mathrm{L}$ sodium citrate) solution for $15 \mathrm{~min}$, and then twice with $0.1 \%$ SDS in $0.2 \times \mathrm{SSC}$ solution for $15 \mathrm{~min}$ at $42^{\circ} \mathrm{C}$. Signals were quantified using a Molecular Imager FX (Bio-Rad, Hercules, CA, USA).

\section{RT-PCR and real-time PCR analysis}

Total RNA was exacted from the embryonic axes and cotyledons by using Trizol Reagent (Invitrogen), according to the manufacturer's instructions. First strand cDNA was synthesized with total RNA using the TaKaRa (Shiga, Japan) RNA PCR Kit (AMV) Ver. 3.0. The relative levels of Mn-SOD were measured by RT-PCR using $N$. nucifera $18 \mathrm{~s}$ rRNA as an internal standard. Second-strand cDNA synthesized and PCR amplification with $M n$ SOD gene-specific primers (forward primer: 5'-CCATCACCAGAAGCACCATC-3'; reverse primer: 5'-TTTAACCACGCCTAGCCACA-3') were carried out using the following conditions: 28 cycles of denaturaton at $94{ }^{\circ} \mathrm{C}$ for $30 \mathrm{~s}$, annealing at $58^{\circ} \mathrm{C}$ for $1 \mathrm{~min}$ and extension at $68{ }^{\circ} \mathrm{C}$ for $1.5 \mathrm{~min}$. The amplification of the internal standard 18s rRNA cDNA was carried out in a similar manner, but with 22 cycles using two 18s rRNA-specific primers (forward primer: 5'-CCATAAACGATGCCGAC-3', reverse primer: 5'CACCACCCATAGAATCAAGA-3'). RT-PCR product was separated on a $2 \%$ agarose gel and visualized by ethidium bromide staining. The expected size of RT-PCR product of the NnMSD1 gene was 325 bp. Real-time PCR was carried out and analyzed with an IQ5 Multicolor Real time PCR Detection System (Bio-Rad) following the manufacturer's instructions with another NnMSD1 gene-specific primer (forward primer: 5'-TTATGCGGCTCCATCACCA-3'; reverse primer: 5'TAACCACTGCGGACGAATC- $3^{\prime}$ ). The program was $95^{\circ} \mathrm{C}$ denaturation for $30 \mathrm{~s}, 40$ cycles of $95^{\circ} \mathrm{C}$ for $10 \mathrm{~s}, 56^{\circ} \mathrm{C}$ for $15 \mathrm{~s}$, and $72{ }^{\circ} \mathrm{C}$ for $20 \mathrm{~s}$.

\section{Acknowledgements}

The authors thank Tara Rintoul for editorial reading of the manuscript.

\section{References}

Akkapeddi AS, Shin DI, Stanek MT, Karnosky DF, Podila GK (1994). cDNA and derived amino acid sequence of the chloroplastic copper/zinc-superoxide dismutase from aspen (Populus tremuloides). Plant Physiol. 106, 1231-1232.

Altschul SF, Gish W, Miller W, Myers EW, Lipman DJ (1990). Basic local alignment search tool. J. Mol. Biol. 215, 403-410.

Bagnoli F, Giannino D, Caparrini S, Camussi A, Mariotti D, Racchi M (2002). Molecular cloning, characterisation and expression of a manganese superoxide dismutase gene from peach (Prunus persica L. Batsch). Mol. Genet. Genomics 267, 321-328.

Baum JA, Scandalios JG (1981). Isolation and characterization of the cytosolic and mitochondrial superoxide dismutase of maize. Arch. Biochem. Biophys. 206, 249-264.

Borgstahl GE, Parge HE, Hickey MJ, Beyer WF, Hallewell RA, Tainer JA (1992). The structure of human mitochondrial manganese superoxide dismutase reveals a novel tetrameric interface of two 4helix bundles. Cell 71, 107-118.

Bowler C, Alliotte TM, De Loose M, Montagu MD, Inze D (1989). The induction of manganese superoxide dismutase in response to stress in Nicotiana plumbaginifolia. EMBO J. 8, 31-38.

Bowler C, Camp W, Montagu M, Inze D (1994). Superoxide dismutase in plants. Cri. Rev. Plant Sci. 13, 199-218.

Breusegem F, Slooten L, Stassart J, Botterman J, Moens T, Montagu M et al. (1999). Effects of overproduction of tobacco MnSOD in maize chloroplasts on foliar tolerance to cold and oxidative stress. J. Exp. Bot. 50, 71-78.

Cannon RE, White JA, Scandalios JG (1987). Clonging of cDNA for maize superoxide dismutase (SOD-2). Proc. Natl. Acad. Sci. USA 84, 179-183.

Carlioz A, Touati D (1986). Isolation of superoxide dismutase mutants in Escherichia coli: is superoxide dismutase necessary for aerobic life? EMBO J. 5, 623-630.

Chen HY, Hu RG, Wang BZ, Chen WF, Liu WY, Schroeder W et al. (2002). Structural studies of an eukaryotic cambialistic superoxide dismutase purified from the mature seeds of camphor tree. Arch. Biochem. Biophys. 404, 218-226. 
Emanuelsson O, Nielsen H, Brunak S, von Heijne G (2000). Predicting subcellular localization of proteins based on their $\mathrm{N}$-terminal amino acid sequence. J. Mol.Biol. 300, 1005-1016.

Fadzilla NM, Finch RP, Burdon RH (1997). Salinity, oxidative stress and antioxidant responses in shoot cultures of rice. J. Exp. Bot. 48, 325-331.

Fink RC, Scandalios JG (2002). Molecular evolution and structure: function relationships of the superoxide dismutase gene families in angiosperms and their relationship to other eukaryotic and prokaryotic superoxide dismutases. Arch. Biochem. Biophys. 399, 19-36.

Fridovich I (1989). Superoxide dismutases: an adaptation to a paramagnetic gas. J. Biol. Chem. 264, 7761-7764.

Hernández JA, Jiménez A, Sevilia F, Mullineaux P (2000). Tolerance of pea (Pisum sativum L.) to long-term salt stress is associated with induction of antioxidant defences. Plant Cell Environ. 23, 853862.

Huang SZ, Tang XJ, Zhang L, Fu JR (2003). Thermotolerance and activity of antioxidative enzymes in lotus seeds. J. Plant Physiol. Mol. Biol. 29, 421-424.

Huang SZ, Tang XJ, Lu CB, Zhang L, Fu JR (2000). Characteristic of superoxide dismutase in lotus seeds. Acta Phytophysiol. Sin. 26, 492-496.

Hwang CS, Baek YU, Yim HS, Kang SO (2003). Protective roles of mitochondrial manganese-containing superoxide dismutase against various stresses in Candida albicans. Yeast 20, 929-941.

Kaminaka H, Morita S, Tokumoto M, Yokoyama H, Masumura T, Tanaka K (1999). Molecular cloning and characterization of a cDNA for an iron-superoxide dismutase in rice (Oryza sativa L.). Biosci. Biotechnol. Biochem. 63, 302-308.

Kliebenstein DJ, Dietrich RA, Martin AC, Last RL, Dangl JL (1999). LSD1 regulates salicylic acid induction of copper zinc superoxide dismutase in Arabidopsis thaliana. Mol. Plant Microbe Interact. 12, 1022-1026.

Lee DH, Kim YS, Lee CB (2001). The inductive responses of the antioxidant enzymes by salt stress in the rice (Oryza sativa L.). J. Plant Physiol. 158, 737-745.

McKersie BD, Chen Y, Beus MD, Bowley SR, Bowler C, Inze D et al. (1993). Superoxide dismutase enhances tolerance of freezing stress in transgenic alfalfa (Medicago sativa L.). Plant Physiol. 103, 11551163.

Nielsen H, Engelbrecht J, Brunak S, von Heijne G (1997). Identification of prokaryotic and eukaryotic signal peptides and prediction of their cleavage sites. Protein Eng. 10, 1-6.

Orr WC, Sohal RS (1994). Extension of life span by overexpression of superoxide dismutase and catalase in Drosophila melanogaster. Science 263, 1128-1130.

Perl-Treves R, Nacmias B, Aviv D, Zeelon EP, Galun E (1988). Isolation of two cDNA clones from tomato containing two different superoxide dismutase sequences. Plant Mol. Biol. 11, 609623.
Parida AK, Das AB, Mohanty P (2004). Defense potentials to $\mathrm{NaCl}$ in a mangrove, Bruguiera parviflora: differential changes of isoforms of some antioxidative enzymes. J. Plant Physiol. 161, 531-542.

Pich U, Schubert I (1993). Midiprep method for isolation of DNA from plants with a high content of polyphenolics. Nucleic Acids Res. 21, 3328.

Privalle CT, Fridovich I (1987). Induction of superoxide dismutase in Escherichia coli by heat shock. Proc. Natl. Acad. Sci. USA 84, 27232726.

Raychaudhuri SS, Deng XW (2000). The role of superoxide dismutase in combating oxidative stress in higher plants. Bot Rev. 66, 89-98.

Schatz G, Krebs H (1987). Signals guiding proteins to their correct locations in mitochondria. Eur. J. Biochem. 165, 1-6.

Shen-Miller J, Schopf JW, Harbottle G, Cao RJ, Ouyang S, Zhou KS et al. (2002). Long-living lotus: germination and soil gammairradiation of centuries-old fruits, and cultivation, growth, and phenotypic abnormalities of offspring. Am. J. Bot. 89, 236-247.

Shin SY, Lee HS, Kwon SY, Kwon ST, Kwak SS (2005). Molecular characterization of a cDNA encoding copper/zinc superoxide dismutase from cultured cells of Manihot esculenta. Plant Physiol. Biochem. 43, 55-60.

Stone $\mathbf{P}$ (2001). The effects of heat stress on cereals yield and quality. In: Basra AS, eds. Crop Responses and Adaptation to Temperature Stress. Food Products Academic Press, New York. pp. 243-291.

Sun J, Folk D, Bradley TJ, Tower J (2002). Induced overexpression of mitochondrial Mn-superoxide dismutase extends the life span of adult Drosophila melanogaster. Genetics 161, 661-672.

Thompson JD, Higgins DG, Gibson TJ (1994). CLUSTAL W: improving the sensitivity of progressive multiple sequence alignment through sequence weighting, position-specific gap penalties and weight matrix choice. Nucleic Acids Res. 22, 4673-4680.

Tsang EWT, Bowler C, Herouart D, Camp W, Villarroel R, Genetello C et al. (1991). Differential regulation of superoxide dismutases in plants exposed to environmental stress. Plant Cell 3, 783-792.

Ushimaru T, Kanematsu S, Katayama M, Tsuji H (2001). Antioxidative enzymes in seedlings of Nelumbo nucifera germinated under water. Physiol. Plant 112, 39-46.

Wang Y, Ying Y, Chen J, Wang X (2004). Transgenic Arabidopsis overexpressing Mn-SOD enhanced salt-tolerance. Plant Sci. 167, 671-677.

Wu G, Wilen RW, Robertson AJ, Gusta LV (1999). Isolation, chromosomal localization, and differential expression of mitochondrial manganese superoxide dismutase and chloroplastic copper/zinc superoxide dismutase genes in wheat. Plant Physiol. 120, 513-520.

Yu Q, Rengel Z (1999). Drought and salinity differentially influenced activities of superoxide dismutases in narrow-leafed lupins. Plant Sci. 142, 1-11.

Zhu D, Scandalios JG (1994). Differential accumulation of manganesesuperoxide dismutase transcripts in maize in response to abscisic acid and high osmoticum. Plant Physiol. 106, 173-178. 